\title{
Modelado de la intención emprendedora con redes bayesianas
}

\section{Modelling entrepreneurial intention with Bayesian networks}

\author{
Jorge López y Ana María Ruiz-Ruano \\ Universidad Católica de Murcia, Murcia, España
}

\begin{abstract}
Resumen
El emprendimiento es considerado hoy en día como un elemento clave en las sociedades postindustriales. Desde un punto de vista psicológico, el emprendimiento puede ser entendido como un proceso actitudinal condicionado por la intención emprendedora. En este trabajo se construyeron, testearon y utilizaron tres modelos de red bayesiana destinados a clarificar las relaciones establecidas entre variables tradicionalmente asociadas al emprendimiento y la intención emprendedora. Una muestra de 254 estudiantes universitarios (73 hombres y 179 mujeres), con edades comprendidas entre los 18 y los 50 años $(\mathrm{M}=$ 22.47, DT $=4.11$ ), rellenó una encuesta destinada a registrar parámetros asociados con la actitud y la intención emprendedora. Con sus respuestas se construyeron tres modelos de red bayesiana que fueron validados y utilizados para realizar predicciones sobre las variables implicadas en el análisis. Los resultados muestran que la deseabilidad percibida, la viabilidad percibida, el locus de control y la influencia familiar son las variables que más influencian la intención emprendedora en emprendedores potenciales. La influencia de estas variables sobre la intención emprendedora fue analizada considerando diferentes escenarios y las relaciones detectadas fueron discutidas con relación a planteamientos teórico-prácticos en el contexto de la potenciación de actitudes emprendedoras.
\end{abstract}

Palabras clave: emprendimiento, educación superior, intención, actitudes, redes bayesianas.

\begin{abstract}
Entrepreneurship is nowadays considered as a key element in postindustrial societies. From a psychological point of view, entrepreneurship can be understood as an attitudinal process conditioned by entrepreneurial intention. We built, tested and used three Bayesian models designed to clarify the relationships between variables commonly associated with entrepreneurship and entrepreneurial intention. A sample of 254 undergraduate university students (73 male and 179 female), ages ranging from 18 to $50(M=22.47, S D=4.11)$, filled in a questionnaire that registered parameters associated with entrepreneurial attitude and intention. Survey answers were used to build three Bayesian networks models which were validated and used to make predictions about the variables involved in the model. Our results show that perceived desirability, perceived feasibility, and the normative social influence derived from the family and the locus of control reliably predict entrepreneurial intention. The influence of these variables over the entrepreneurial intention was analyzed considering different scenarios, and the discovered relationships between variables where discussed from practical and theoretical points of view in the context of promoting entrepreneurial attitudes.
\end{abstract}

Keywords: entrepreneurship, higher education, intention, attitudes, Bayesian nets.

Contacto: J. López. Facultad de Ciencias de la Salud. Departamento de Ciencias de la Salud. Universidad Católica de Murcia. Campus de los Jerónimos s/n, 30107, Guadalupe, Murcia, España.jpuga@ucam.edu

Cómo citar este artículo:

López, J. y Ruiz-Ruano, A. M. (2014). Modelado de la intención emprendedora con redes bayesianas. Revista de Psicología, 23(2), 71-87. http://dx.doi.org/10.5354/0719-0581.2014.36149 


\section{Introducción}

El rol de las personas emprendedoras está ganando protagonismo en las sociedades postindustriales como elemento que favorece el desarrollo social y económico tanto a nivel local como global. Como señaló Shapero (1981) hace más de tres décadas, el modelo económico basado en la explotación de recursos humanos poco cualificados por parte de grandes multinacionales es poco deseable en comparación con economías locales que se autorenuevan y que se sostienen por pequeñas empresas. Este segundo modelo, que tiende a ser más sostenible en el tiempo, es el caldo de cultivo ideal para que las personas emprendedoras pongan en marcha su creatividad para dinamizar el desarrollo social y económico de sus regiones. Por ello, el emprendimiento es considerado como la fuente que "proporciona a las comunidades la diversidad y el dinamismo que no sólo favorece el desarrollo continuo, sino que también proporciona un entorno en el que la libertad personal y los derechos individuales pueden florecer" (Shapero, 1985, p. 5).

Sin embargo, pese a la relevancia que se atribuye al emprendimiento, este sigue siendo un fenómeno social difícil de estudiar y conceptualizar. La analogía introducida por Rogoff y Lee (1996) es muy ilustrativa en este sentido, ya que equiparan el estudio de las partículas subatómicas, por parte de la física, al estudio de las personas emprendedoras desde la óptica de las ciencias sociales. Según los autores, de igual modo que la física observa el comportamiento de las partículas subatómicas pero no puede explicarlo, las ciencias sociales se percatan de los efectos de las personas emprendedoras pero no pueden explicar su comportamiento. En cierto modo, la creación de empresas es algo caótico, desordenado, turbulento y sensible a las condiciones iniciales que propicia cierta ausencia de replicabilidad del fenómeno (Smilor y Feeser, 1991). Por ello, pese a que se han desarrollado modelos orientados a optimizar las probabilidades de desarrollo de nuevas aventuras empresariales (e.g., Bhave, 1994), únicamente un bajo porcentaje de las empresas de nueva creación sobreviven a los cinco primeros años de vida (Fuller-Love, 2006).
Desde una perspectiva psicológica, el modelo de los rasgos ha sido el paradigma predominante hasta mediados de los años ochenta del pasado siglo (e.g., Fuller-Love, 2006; McKenzie, Ugbah y Smothers, 2007; Thompson, 2004). El modelo de los rasgos asume que las personas que crean empresas se diferencian de las demás por un conjunto de rasgos de personalidad relativamente estables que sirven para catalizar la creación de un nuevo negocio. Así, aspectos como una alta motivación de logro (Boyatzis, 1982; Genescá y Capelleras, 2004; McClelland, 1955, 1961; Veciana, 1989), un perfil de locus de control interno (García, Cano y Gea, 2005; Sánchez, 2003; Stanworth, Stanworth, Granger y Blyth, 1989; Welsch y Young, 1982), el optimismo o la tolerancia al riesgo (Cantillon, 1755/2010; Cooper, Woo y Dunkelberg, 1988; Hmieleski y Baron, 2009; Liang y Dunn, 2008, 2010; Lovallo y Kahneman, 2003; Welsch y Young, 1982) son algunos rasgos que han sido asociados a las personas que crean empresas.

Sin embargo, esta perspectiva ha recibido numerosas críticas como paradigma explicativo y predictivo de la creación de empresas (Bird, 1988; Gartner, 1985, 1988). Por ejemplo, Robinson, Stimpson, Huefner y Hunt (1991) apuntaron cuatro grandes bloques de críticas que atacaban la perspectiva de los rasgos como modelo explicativo de los procesos individuales que daban lugar a la creación de empresas. En primer lugar, Robinson et al. (1991) aluden a un problema metodológico, dado que los instrumentos de medida que se venían utilizando en la psicología general se tomaron para el estudio del emprendimiento sin las adaptaciones pertinentes. Por otro lado, la interpretación de las medidas adoleció de los criterios básicos de validez exigibles a cualquier medida de rasgos psicológicos. En tercer lugar, los autores consideran que las teorías de la personalidad surgidas en el seno de la psicología hubiesen merecido la consiguiente adaptación al contexto de la investigación sobre la creación de empresas. Por último, Robinson et al. (1991) consideran que el modelo de los rasgos tendría que haber tenido en consideración las teorías interactivas de la personalidad que se comenzaron a forjar durante el apogeo del citado modelo. 
Un paradigma complementario y, en cierto modo, competidor del modelo de los rasgos para comprender o estudiar el fenómeno emprendedor es el basado en las actitudes. Desde esta otra perspectiva, se defiende que la creación de empresas está mediatizada por un proceso intencional y deliberado que conduce al emprendedor potencial a crear un negocio en interacción constante con el entorno social que le circunda. Por tanto, para este modelo la creación de empresas es fruto tanto de las predisposiciones estables de respuesta en la persona potencialmente emprendedora, como de la interacción que se produce entre la persona con las condiciones sociales y culturales en las que se ve envuelta (cf., Bird, 1988; Krueger y Brazeal, 1994; Krueger y Carsrud, 1993; Krueger, Reilly y Carsrud, 2000; Liñán, Battistelli y Moriano, 2008; Moriano, Gómez, Laguna, y Roznowsky, 2008).

Licht y Siegel (2006) reconocen que el trabajo de Shapero y Sokol (1982) podría considerarse como un punto arquimédico que marcó un antes y un después con relación al estudio de los condicionantes sociales que influyen en la creación de empresas. Para Shapero y Sokol (1982) el mayor condicionante social que influye en la creación de empresas son los valores que una sociedad fomenta o promueve con relación al emprendimiento. Así, en la medida que una cultura o sociedad concreta presenta la creación de empresas como algo viable y deseable, la probabilidad de que una persona potencialmente emprendedora cree una empresa, aumenta. Sin embargo, en esta propuesta está implícita la idea de que cualquier persona no se convierte en emprendedor ineludiblemente cuando considera viable y deseable el desarrollo de una aventura empresarial. Más bien, para estos autores, la persona se convierte en emprendedora como consecuencia de una experiencia negativa caracterizada por una sensación subjetiva de rechazo por parte de la sociedad (Shapero, 1975). En este sentido, la persona que crea una empresa encajaría en el perfil de un individuo que ha sido "marginado" por la sociedad (e.g., Stanworth et al., 1989; Veciana, 1989) pero que, a la vez, considera el emprendimiento como algo deseable y viable.
La propuesta sobre la influencia de la deseabilidad y la viabilidad en la intención emprendedora ha sido testada empíricamente en repetidas ocasiones (e.g., Fitzsimmons y Douglas, 2011; Krueger y Brazeal, 1994; Krueger y Carsrud, 1993; Moriano et al., 2008). Su estudio ha sido realizado bajo la óptica de la Teoría de la Acción Planeada (Ajzen y Fishbein, 2005) entendida como una evolución de la Teoría de la Acción Razonada (Ajzen y Fishbein, 1980; Fishbein y Ajzen, 1975), considerando que la creación de un nuevo negocio es fruto de un proceso intencional mediado por las interpretaciones subjetivas que la persona realiza sobre ciertos elementos sociales.

Un metaanálisis reciente que estudia la integración entre el modelo de la intención emprendedora y la Teoría de la Acción Razonada puede encontrarse en Schlaegel y Koening (2014). Sin embargo, los trabajos destinados a estudiar el fenómeno emprendedor con redes bayesianas o metodología bayesiana son más limitados (e.g., Block, Hoogerheide y Thurik, 2012; López y García, 2012; López, Ramírez y Casado, 2012; Sohn y Lee, 2013) y, por ello, uno de los objetivos de este estudio es mostrar la utilidad de este tipo de técnica estadística para modelar la intención emprendedora. Las redes bayesianas son herramientas de modelado estadístico surgidas en el ámbito de la inteligencia artificial y se caracterizan por representar un aspecto concreto de la realidad, tanto desde un punto de vista cualitativo como cuantitativo (López y García, 2011a). Técnicamente, las redes bayesianas pertenecen a una familia de modelos gráficos destinados a modelar un problema de estudio de un modo altamente estructurado (Cowell, Dawid, Lauritzen y Spiegelhalter, 1999; Martínez y Rodríguez, 2003).

Como se ha indicado con anterioridad, se considera que las dos claves principales que explican la intención emprendedora son la deseabilidad y la viabilidad (Fitzsimmons y Douglas, 2011; Krueger y Brazeal, 1994; Krueger y Carsrud, 1993; Licht y Siegel, 2006; Moriano et al., 2008; Shapero, 1982) y, por consiguiente, predecimos que en un modelo de red bayesiana estas dos variables también serán las que mayor varianza expliquen. Adicionalmente, se compararán tres modelos diferentes de red bayesiana 
con el objetivo de identificar la intensidad de las relaciones que se establecen entre las variables. En concreto, se testará un modelo estructural basado en la teoría actual sobre emprendimiento, un modelo de clasificador ingenuo bayesiano que sería análogo a la regresión logística y un modelo TAN (Tree Augmented NaïveBayes) que se diferencia del clasificador ingenuo en que tolera la presencia de relaciones entre las variables diagnósticas o de clasificación (Greiner, Su, Shen y Zhou, 2005; Greiner y Zhou, 2002; Shen, Su, Greiner, Musilek y Cheng, 2003).

Por consiguiente, este estudio tendrá una doble utilidad: una finalidad teórico-práctica y otra orientada al aspecto metodológico. En cuanto al aspecto teórico-práctico nuestro trabajo servirá para arrojar luz sobre las relaciones del modelo actitudinal que explica la intención de crear una empresa en emprendedores potenciales. En este sentido, este trabajo podría ser de utilidad frente a la orientación que pudiera ofrecerse a los emprendedores potenciales que aspiran a crear su propio negocio (Fitzsimmons y Douglas, 2011). Por ejemplo, los emprendedores potenciales podrían ser informados sobre las variables que más influyen en su intención emprendedora y advertirles sobre aquellos aspectos que pueden afectar negativamente en el futuro desarrollo de su negocio. Una variable que podría considerarse perniciosa frente a la creación de empresas es el exceso de optimismo (e.g., Cooper et al., 1988; Hmieleski y Baron, 2009; Lovallo y Kahneman, 2003). Así, un modelo de red bayesiana que considerase la influencia negativa de esa variable sobre la intención emprendedora podría ser de utilidad para orientar a los futuros emprendedores. En nuestro trabajo, las variables utilizadas que se relacionan con cierta forma de optimismo (diferentes formatos de riesgo y obstáculos percibidos) podrían servir a tal efecto.

Por su parte, este trabajo también presentará las posibilidades que ofrecen las redes bayesianas para modelar un fenómeno psicosocial como el emprendimiento con el consiguiente beneficio que esto podría aportar a la comunidad científica. Así, por ejemplo, el uso de este tipo de herramienta permitirá la representación del modelo estadístico tanto gráfica como cuantitativamente (Edwards, 1998; Heckerman, 1995). Aunque los modelos gráficos que incorporan información cuantitativa han recibido considerable atención en el área de las ciencias sociales por medio de la utilización de modelos de ecuaciones estructurales; también es cierto que, como indican Anderson y Vastag (2004), las redes bayesianas tienen ciertas ventajas frente a este tipo de modelos. La posibilidad de combinar conocimiento previo con datos observados del problema modelado (Nadkarni y Shenoy, 2004), la no asunción de supuestos distribucionales en los datos (Anderson y Vastag, 2004) y el buen funcionamiento de estas técnicas con muestras pequeñas (López y García, 2011b) son ventajas que se aplican al caso particular que estamos abordando.

\section{Participantes}

La muestra estuvo compuesta por 254 estudiantes universitarios. El 28.97\% fueron hombres (73) y el $71.03 \%$ mujeres (179), cuyas edades oscilaron entre los 18 y 50 años $(M=$ 22.47 ; DT $=4.11)$. El $47.22 \%$ de los participantes se encontraban cursando estudios de Psicología (119), el 25.79\% Licenciatura en Administración y Dirección de Empresas (LADE) (65), el $13.89 \%$ empresariales (35) y el $13.1 \%$ Magisterio (33). Del total de participantes, 76 se encontraban entre el primer y segundo curso de la carrera $(30.16 \%), \quad 66$ cursaban tercero $(26.19 \%), 80$ se encontraban cursando cuarto $(31.75 \%)$ y 30 se encontraban en el último curso de la carrera $(11.90 \%)$.

\section{Instrumentos}

Para recoger los datos se diseñó una encuesta electrónica con la plataforma LimeSurvey (LimeSurvey Project Team, 2013). La encuesta estuvo integrada por cuatro secciones. En la primera sección se recogieron los datos sociodemográficos necesarios para caracterizar la muestra de participantes. En la sección segunda se presentaron las escalas de locus de control y riesgo percibido. La tercera sección contuvo preguntas relacionadas con los modelos actitudinales de emprendimiento $\mathrm{y}$, por último, la cuarta sección se utilizó para que los participantes informasen voluntariamente de sus datos de 
contacto para participar en fases posteriores de la investigación.

Para medir el locus de control emprendedor se utilizó la escala de Moriano et al. (2008). Esta escala contiene tres ítems puntuables en una escala tipo Likert con siete alternativas (1 a 7) en los que el participante ha de expresar el grado de interés que le suscitan conductas típicamente asociadas a un perfil cognitivo de persona emprendedora, caracterizada por un locus de control interno. El índice de consistencia interna observado en nuestro estudio es de $.7\left(\mathrm{IC}_{\text {sup }} 90 \%=.75, \mathrm{IC}_{\text {inf }} 90 \%=.64\right)$, pero en estudios previos esta escala mostró una consistencia interna de .77 (Moriano et al., 2008).

Por su parte, para valorar el riesgo asociado a la creación de empresas los participantes fueron instados a responder tres ítems tipo Likert con cuatro alternativas de respuesta indicando el grado en que el contenido de cada ítem suscitaba riesgo percibido (desde nada o casi nada a mucho). Cada uno de los tres ítems estuvo referido al riesgo asociado a la actividad económica de la empresa, al riesgo económico personal y al riesgo para la carrera profesional personal. Los ítems no se agregaron en una única puntuación referida al riesgo global percibido, pero las respuestas que dieron los participantes a cada ítem se recodificaron en dos grupos: las dos opciones más altas de respuesta para indicar riesgo percibido y las opciones más bajas de respuesta para indicar ausencia de riesgo percibido.

La mayor parte de las variables destinadas a medir componentes actitudinales fueron presentadas a los participantes de forma dicotómica. La intención emprendedora se evaluó con la pregunta: ¿Has pensado seriamente en crear tu propia empresa?, cuyas alternativas de respuesta fueron sí y no. La deseabilidad de crear una empresa también se valoró con una escala de respuesta dicotómica con las alternativas $s i ́$ y no ante la pregunta: ¿Consideras deseable crear una empresa propia al finalizar tus estudios? Del mismo modo, se introdujeron dos preguntas para valorar la influencia de las normas sociales utilizando el mismo esquema de respuesta: una para considerar la influencia de la familia
(¿Le gustaría a tu familia que creases tu propio negocio?) y otra para estudiar la influencia de los iguales (iLe gustaría a tus amigos que creases tu propio negocio?). La actitud de los participantes hacia la creación de empresas se midió con la pregunta: ¿Crees que la creación de empresas es algo positivo o negativo?, donde las opciones de respuesta fueron algo positivo y algo negativo. La cantidad de obstáculos percibidos también fue valorado con una pregunta dicotómica (iQué cantidad de obstáculos crees que encontrarias si decidieses crear tu propio negocio?) en la que las alternativas de respuesta fueron muchos y pocos. Por último, la viabilidad de la creación de empresas se valoró a tres niveles: desde un punto de vista general, desde el punto de vista de la viabilidad de oportunidades y desde el punto de vista de la viabilidad entendida como recursos económicos (Cohen y Winn, 2007; McMullen y Shepherd, 2006; Shapero, 1981).

La viabilidad (general) se midió utilizando un ítem tipo Likert con cuatro alternativas de respuesta que demandaba que el participante indicase el grado de viabilidad que percibía con relación a la creación de una empresa propia (desde muy poco viable a muy viable). Por su parte, la viabilidad de recursos (¿Qué importancia crees que tiene la disponibilidad de recursos (económicos, contactos, conocimientos, etc.) frente a la decisión de crear una empresa?) y la viabilidad de oportunidad (¿Qué importancia crees que tiene la detección de una oportunidad de negocio (una idea innovadora, detección de necesidades, nuevo método de producción, etc.) frente a la decisión de crear una empresa?) se evaluaron con tres alternativas de respuesta que hacían referencia a la importancia atribuida a cada aspecto de la viabilidad (ninguna, alguna y mucha).

\section{Procedimiento}

Para acceder a los participantes se diseñó un mensaje de correo electrónico de invitación que contenía una explicación de los objetivos de la investigación y el hiperenlace al formulario electrónico correspondiente. El mensaje de invitación se envió haciendo uso de la herramienta de correo electrónico integrada en la plataforma de enseñanza virtual que los alumnos utilizaban para seguir sus asignaturas de la universidad. Los participantes no recibieron 
ningún tipo de compensación económica ni crediticia por participar en la investigación y la única gratificación que recibieron por responder al cuestionario electrónico fue de carácter verbal.

\section{Análisis de datos}

La especificación de las estructuras gráficas fueron determinadas como pasos previos a la estimación paramétrica (Cowell et al., 1999). Se consideraron tres modelos gráficos: uno basado en la teoría existente en la literatura, otro basado en criterios metodológicos (el clasificador ingenuo, o simple, bayesiano) $y$, por último, uno basado en criterios estrictamente estadísticos (el TAN, Tree Augmented Naïve-Bayes o clasificador bayesiano optimizado). El modelo teórico testado en este estudio aparece representado en la figura 1, donde queda reflejado que la intención se relaciona con la viabilidad y la deseabilidad. Por su parte, la deseabilidad estaría asociada a tres tipos de riesgo (actividad de la empresa, económico personal y de carrera profesional), a la actitud hacia la creación de empresas y a las normas sociales (tanto desde el punto de vista familiar como desde la óptica de la interacción con los iguales). Por su parte, la viabilidad estaría asociada a dos componentes de la viabilidad (enfocados a los recursos económicos y a la oportunidad de negocio), a los obstáculos percibidos y al locus de control.
El modelo TAN se estimó con el algoritmo implementado en la versión 5.12 de Netica (Norsys Software Corp., 2013), mientras que el clasificador simple bayesiano, dada su similitud con la regresión logística (Greiner et al., 2005; Greiner y Zhou, 2002; Shen et al., 2003), queda definido por la variable a predecir, antecedente o madre (en nuestro caso la intención emprendedora) y un conjunto de variables predictoras o hijas (el resto de variables implicadas en el estudio) y mostradas en la figura 1.

La estimación paramétrica se llevó a cabo utilizando el procedimiento de máxima verosimilitud corregido con la ley de la sucesión de Laplace (Greiner et al., 2005). Para valorar la bondad de ajuste de los modelos se utilizaron parámetros de validación predictiva (López y García, 2011a), la pérdida logarítmica, la pérdida cuadrática y la compensación esférica (Pearl, 1978). Por último, para indagar en la influencia que tenía cada variable sobre el nodo predicho, se llevó a cabo un análisis de sensibilidad basado en el porcentaje de influencia sobre este nodo (Neapolitan, 1990). El resto de análisis de datos fue realizado utilizando la versión 3.1.2 del software de computación estadística R (R Core Team, 2014).

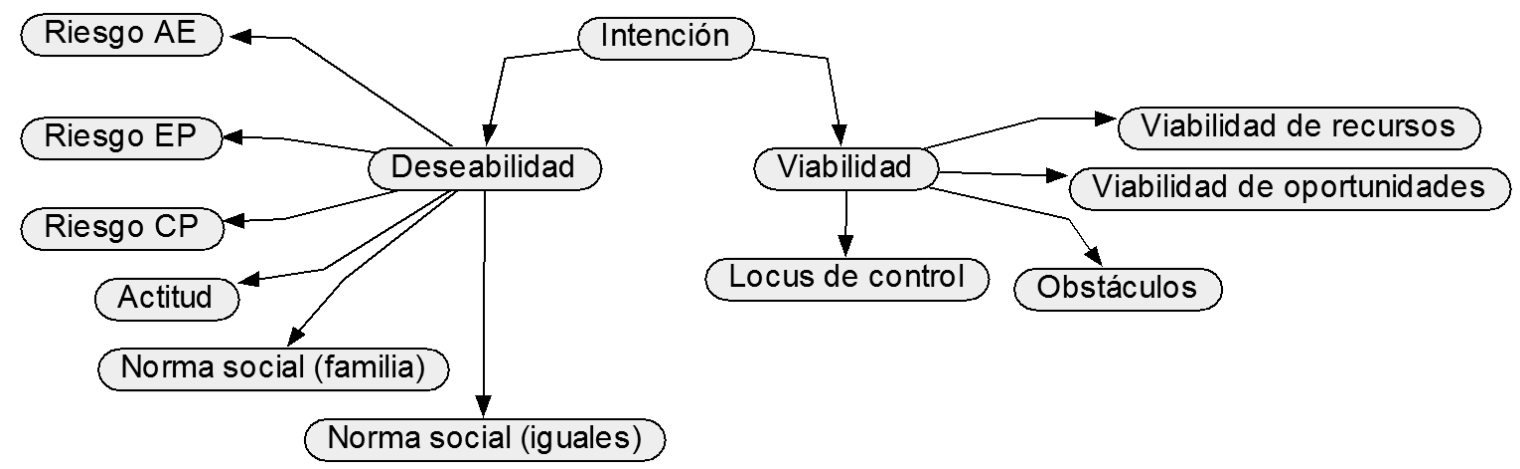

Figura 1. Modelo de red bayesiana teórica. Riesgo AE: riesgo asociado a la actividad económica de la empresa; Riesgo EP: riesgo económico personal; Riesgo CP: riesgo en la carrera profesional. 


\section{Resultados}

La tabla 1 muestra que la deseabilidad, la viabilidad, la norma social vinculada a los iguales y la viabilidad de oportunidades, se asocian positivamente a la intención emprendedora. En lo que respecta al locus de control, los participantes de nuestro estudio que mostraron una mayor intención emprendedora se caracterizan por mayor locus de control interno $(M=13.97, S D=3.99)$ si los comparamos con los que muestran menor intención emprendedora $(M=12.08, S D=$ 3.17), $t(218.70)=3.98, p<.001, d=0.52,1-\beta=$ .99 , contraste unilateral.

Como se puede apreciar en la figura 2, el algoritmo TAN genera un modelo de red bayesiana que contiene 11 enlaces adicionales al modelo basal definido por el clasificador simple baye- siano. Es destacable señalar que la deseabilidad global de la creación de una empresa aparece condicionada a la viabilidad percibida, mientras que esta segunda se muestra dependiente de las normas sociales derivadas del contexto familiar. La actitud hacia la creación de empresas también se muestra como dependiente de la viabilidad percibida y la influencia normativa del grupo de iguales aparece como dependiente del contexto social familiar. Es también destacable que el modelo identifica que la relación que se establece entre viabilidad y obstáculos percibidos estaría mediada por el locus de control. Según el modelo estimado por el TAN, la viabilidad entendida desde el punto de vista de la detección de oportunidades empresariales influiría sobre las normas sociales familiares y sobre la viabilidad de recursos. Y, por último, dos de estos enlaces

Tabla 1

Frecuencias observadas (porcentajes entre paréntesis) para cada nivel de la variable y su relación con la intención emprendedora

\begin{tabular}{lllrr}
\hline & Alto/a & Bajo/a & $\chi^{2 \mathrm{~b}}$ & $p$ \\
\hline Intención & $116(50.22)$ & $115(49.78)$ & & \\
Riesgo AE & $207(87.71)$ & $29(12.29)$ & 1.36 & .24 \\
Riesgo EP & $197(83.47)$ & $39(16.53)$ & 1.05 & .31 \\
Riesgo CP & $128(54.24)$ & $108(45.76)$ & 0.22 & .64 \\
Actitud & $226(97.84)$ & $5(2.16)$ & 3.31 & .07 \\
NS (familia) & $188(81.39)$ & $43(18.61)$ & 1.09 & .30 \\
NS (iguales) & $206(89.18)$ & $25(10.18)$ & 14.71 & $<.01$ \\
Deseabilidad & $126(54.55)$ & $105(45.45)$ & 48.04 & $<.01$ \\
Viabilidad & $134(58.01)$ & $97(41.99)$ & 21.05 & $<.01$ \\
Viabilidad Ra & $204(88.31)$ & $27(11.69)$ & 0.15 & .67 \\
Viabilidad Oa & $192(83.12)$ & $39(16.89)$ & 8.06 & $<.01$ \\
Obstáculos & $202(87.45)$ & $29(12.55)$ & 2.44 & .12 \\
Sexo & $73(28.97-\mathrm{H})$ & $179(71.03-\mathrm{M})$ & 2.02 & .15 \\
\hline
\end{tabular}

Nota. Riesgo AE: riesgo asociado a la actividad económica de la empresa; Riesgo EP: riesgo económico personal; Riesgo CP: riesgo en la carrera profesional; NS: norma social; Viabilidad R: viabilidad de recursos; Viabilidad O: viabilidad de oportunidades; $\mathrm{H}$ : hombre, M: mujer.

${ }^{a}$ Estas variables se recodificaron en alta y baja dado que la alternativa de respuesta más baja fue elegida en una proporción muy pequeña de participantes.

b Los grados de libertad para las $\chi^{2}$ es 1 en todos los casos. 
identificados por el algoritmo TAN generan un camino dirigido entre el riesgo asociado a la actividad económica y el riesgo asociado a la continuidad de la carrera profesional, pasando por el riesgo vinculado a la economía personal.

La red que mejor predice la intención emprendedora es la generada automáticamente por el algoritmo TAN (tabla 2), que mejora en un 3.9\% la clasificación correcta de casos que realiza el clasificador simple y en un $4.7 \%$ respecto de lo que lo hace la red teórica. La red TAN también es más sensible que los otros dos modelos al detectar la intención emprendedora, pero el clasificador simple tiene un mayor grado de especificidad. De manera general, el modelo generado automáticamente se comporta ligeramente mejor al tratar de predecir la intención emprendedora frente al modelo teórico y al modelo de clasificación simple. Sin embargo, no existen diferencias estadísticamente significativas entre la tasa de clasificaciones correctas realizadas por el modelo TAN cuando la comparamos con la de la red teórica, $\chi^{2}(1)=2.745, p=.10$, contraste bilateral o con el clasificador simple bayesiano, $\chi^{2}(1)=1.795, p=.18$, contraste bila- teral. Además, este incremento predictivo se produce a costa de una reducción en la parsimonia del modelo, entendida como el número de parámetros y de enlaces en el modelo. Así, mientras que tanto la red teórica como el clasificador simple bayesiano son capaces de realizar sus predicciones con 52 parámetros, el modelo TAN necesita más del doble para predecir la intención emprendedora. Del mismo modo, tanto el modelo teórico como el clasificador simple tienen 12 enlaces, mientras que la red TAN necesita casi el doble (24).

Aunque podemos observar diferencias relativas en la influencia que ejerce cada variable sobre la intención emprendedora en función del modelo que estemos utilizando, todos los modelos coinciden al identificar que las dos variables que más influyen sobre la intención son la deseabilidad y la viabilidad (tabla 3). Adicionalmente, las otras dos variables que más variabilidad explican de la intención emprendedora son la influencia normativa de los iguales (amigos) y el locus de control. No obstante, el orden de importancia de estas variables depende de si utilizamos el modelo TAN, el teórico o el clasificador simple.

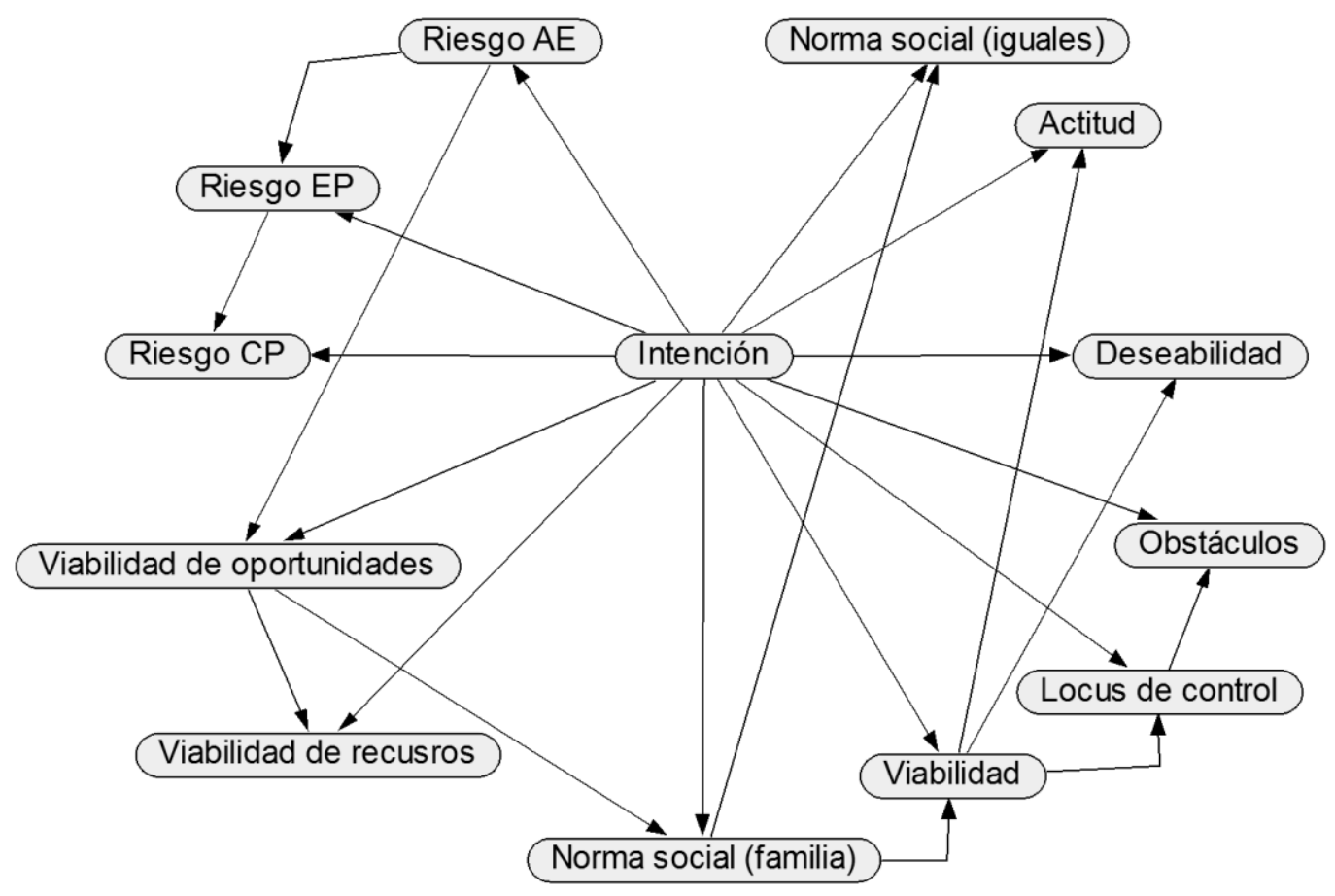

Figura 2. Modelo de red bayesiana TAN estimado. Riesgo AE: riesgo asociado a la actividad económica de la empresa; Riesgo EP: riesgo económico personal; Riesgo CP: riesgo en la carrera profesional. 
Tabla 2

Índices de bondad de ajuste de los modelos

\begin{tabular}{lccc}
\hline & $\begin{array}{c}\text { Red } \\
\text { TAN }\end{array}$ & $\begin{array}{c}\text { Red } \\
\text { teórica }\end{array}$ & $\begin{array}{c}\text { Clasifica- } \\
\text { dor } \\
\text { simple }\end{array}$ \\
\hline Enlaces & 23 & 12 & 12 \\
Parámetros & 106 & 52 & 52 \\
Sensibilidad & .819 & .776 & .681 \\
Especificidad & .739 & .687 & .800 \\
PFP & .261 & .313 & .200 \\
PFN & .181 & .224 & .319 \\
VPP & .760 & .714 & .775 \\
VPN & .802 & .752 & .713 \\
TCC & .779 & .732 & .740 \\
$\begin{array}{l}\text { Pérdida } \\
\text { logarítmica }\end{array}$ & .475 & .563 & .543 \\
$\begin{array}{l}\text { Pérdida } \\
\text { cuadrática }\end{array}$ & .313 & .377 & .362 \\
$\begin{array}{l}\text { Compensa- } \\
\text { ción esférica }\end{array}$ & .825 & .789 & .797 \\
\hline
\end{tabular}

Nota: PFP: proporción de falsos positivos; PFN: proporción de falsos negativos; VPP: valor predictivo positivo; VPN: valor predictivo negativo; TCC: tasa de clasificaciones correctas.

Las predicciones que hacen los tres modelos van en la misma dirección cuando consideramos la influencia secuencial de las cuatro variables que más impacto tienen sobre los mismos. En la tabla 4 aparecen representados, para cada uno de los modelos, los cambios que se producen en los niveles estimados de intención emprendedora cuando consideramos un escenario favorable (una respuesta positiva a cada una de las variables predictoras) y desfavorable (una respuesta negativa a cada una de las variables predictoras) a la intención emprendedora. Como se puede observar, la intención emprendedora aumenta entre un $17.8 \%$ (modelo TAN) y un $20.6 \%$ (clasificador simple) cuando se observa una alta deseabilidad en un estudiante universitario. Por el contrario, cuando la deseabilidad es baja, la intención emprendedora se reduce entre un
$24.5 \%$ (modelo TAN) y un $28 \%$ (clasificador simple). Si, adicionalmente, consideramos que la viabilidad percibida es alta, la probabilidad de que un estudiante universitario experimente una alta intención emprendedora aumenta entre un $28.1 \%$ (modelo TAN) y un 30.5\% (modelo teórico). De manera antagónica, cuando la deseabilidad y la viabilidad son bajas, la probabilidad de que un estudiante manifieste intención de crear una empresa desciende entre un 29.5\% (modelo TAN) y un $37.7 \%$ (clasificador simple).

\section{Discusión y conclusiones}

En este trabajo hemos desarrollado, testado y utilizado tres modelos de red bayesiana para modelar la intención emprendedora en una muestra de estudiantes universitarios. Los tres modelos coinciden en indicar que la deseabilidad y la viabilidad son los predictores más importantes de la intención emprendedora. Por consiguiente, nuestros resultados apuntan en la misma línea trazada por los estudios previos que han sugerido, basándose en las ideas de Shapero y Shokol (1982), que la deseabilidad y la viabilidad son variables clave para entender el comportamiento de la intención emprendedora (Fitzsimmons y Douglas, 2011; Krueger y Brazeal, 1994; Krueger y Carsrud, 1993; Moriano et al., 2008). Además de la deseabilidad y la viabilidad, los tres modelos desarrollados y testados han mostrado que tanto la influencia de los iguales (amigos normalmente en este nivel educativo) como el locus de control son también variables relevantes para comprender la naturaleza de la intención emprendedora. Dado que las redes bayesianas son instrumentos estadísticos susceptibles de ser utilizados para orientar u optimizar la toma de decisiones (e.g., Edwards y Fasolo, 2001; López y García, 2011a), los modelos aquí desarrollados podrían ser de utilidad en el contexto aplicado para diseñar políticas o estrategias destinadas a la potenciación del emprendimiento entre los estudiantes universitarios. Así, como indican Smilor y Feeser (1991), herramientas como las que hemos desarrollado en este trabajo podrían ser de utilidad en la gestión del emprendimiento a nivel universitario donde, normalmente, se comienzan a desarrollar iniciativas de creación de empresas que, presumiblemente, tendrán efectos positivos en el tejido productivo. 
Tabla 3

Porcentaje de influencia que ejerce cada variable sobre la intención emprendedora en cada modelo

\begin{tabular}{lccc}
\hline Nodos & $\begin{array}{c}\text { Red } \\
\text { TAN }\end{array}$ & $\begin{array}{c}\text { Red } \\
\text { Teórica }\end{array}$ & $\begin{array}{c}\text { Clasificador } \\
\text { Simple }\end{array}$ \\
\hline Deseabilidad & $15.60(1)$ & $15.60(1)$ & $15.00(1)$ \\
Viabilidad & $6.68(2)$ & $6.84(2)$ & $4.88(2)$ \\
Norma social (iguales) & $4.66(3)$ & $0.49(4)$ & $2.14(4)$ \\
Locus de control & $3.61(4)$ & $0.61(3)$ & $3.14(3)$ \\
Viabilidad de oportunidades & $2.66(5)$ & $0.16(5)$ & $1.71(5)$ \\
Actitud & $0.94(6)$ & $0.02(9)$ & $0.08(10)$ \\
Obstáculos & $0.84(7)$ & $0.11(7)$ & $0.06(11)$ \\
Riesgo AE & $0.47(8)$ & $0.00(12)$ & $0.54(7)$ \\
Norma social (familia) & $0.42(9)$ & $0.14(6)$ & $0.21(9)$ \\
Riesgo EP & $0.36(10)$ & $0.05(8)$ & $1.38(6)$ \\
Riesgo CP & $0.10(11)$ & $0.00(11)$ & $0.44(8)$ \\
Viabilidad de recursos & $0.08(12)$ & $0.02(10)$ & $0.02(12)$ \\
\hline
\end{tabular}

Nota: Riesgo AE: riesgo asociado a la actividad económica de la empresa; Riesgo EP: riesgo económico personal; Riesgo CP: riesgo en la carrera profesional. Los números entre paréntesis indican la posición de influencia relativa que ocupa cada variable en un modelo concreto.

Como se puede apreciar en la tabla 4, el cambio que experimenta la intención emprendedora cuando conocemos el estado de las cuatro variables más influyentes en cada uno de los modelos (deseabilidad, viabilidad, influencia social normativa de los iguales y locus de control) es sustancial. Para el modelo TAN y para el clasificador simple bayesiano, la probabilidad de que la intención emprendedora sea alta cuando se dan respuestas favorables a estas cuatro variables, aumenta entre un 32 y un 34 por ciento, respectivamente. A su vez, la reducción que se produce en la probabilidad de que la intención emprendedora sea alta cuando nos ponemos en el peor de los casos para las cuatro variables mencionadas, oscila entre un 45 y un 48 por ciento para los modelos TAN y de clasificación simple, respectivamente. Estos datos son más moderados si tenemos en cuenta el modelo teórico planteado inicialmente (en torno a un $30 \%$ de aumento cuando nos posicionamos en un escenario favorable y una reducción del 36\% cuando suponemos el peor de los casos).
Estos resultados sugieren pautas estratégicas de acción que podrían orientarse a potenciar la intención emprendedora entre los estudiantes universitarios. Así, si se tratase de mostrar que la creación de una empresa propia es algo deseable para la sociedad y viable desde el punto de vista financiero, numerosos estudiantes universitarios podrían ver esta salida laboral como algo plausible al terminar sus estudios, del mismo modo a como lo ve la población general (Fitzsimmons y Douglas, 2011; Krueger y Brazeal, 1994). Así, de la misma manera a como han hecho notar Dreisler, Blenker y Nielsen (2003) para el caso danés, se podrían desarrollar programas de intervención orientados a estimular actitudes positivas hacia la creación de empresas en los estudiantes universitarios o, como señalan Liñan, Rodríguez-Cohard y RuedaCantuche (2011), comenzar a trabajar la orientación emprendedora a niveles preuniversitarios. 
Tabla 4

Cambio secuencial que experimenta la intención emprendedora en función de un escenario favorable (deseabilidad, viabilidad, influencia social y locus de control altos) o desfavorable (deseabilidad, viabilidad, influencia social y locus de control bajos)

\begin{tabular}{llcccc}
\hline Escenario & Modelo & Deseabilidad & Viabilidad & $\begin{array}{c}\text { Norma social } \\
\text { (familia) }\end{array}$ & $\begin{array}{c}\text { Locus de } \\
\text { control }\end{array}$ \\
\hline Favorable & Red TAN & +20.6 & +28.1 & +30.0 & +34.4 \\
& Red Teórica & +19.9 & +30.5 & & \\
& Clasificador & +17.8 & +25.9 & +27.4 & +32.3 \\
& Simple & & & & \\
Desfavorable & Red TAN & -24.5 & -29.5 & -45.0 & -45.5 \\
& Red Teórica & -25.0 & -36.4 & & \\
& Clasificador & -28.0 & -37.7 & -47.4 & -48.6 \\
& Simple & & & & \\
\hline
\end{tabular}

Nota: La probabilidad previa de que un estudiante muestre intención emprendedora es del $50.2 \%$.

Por otro lado, el modelo generado automáticamente (TAN) ha mostrado relaciones entre variables que deberían de ser estudiadas con más detenimiento en futuros estudios. Cabría destacar algunas relaciones interesantes que han sido puestas de manifiesto en la investigación sobre este fenómeno. Por un lado, se observa que tanto la actitud hacia la creación de empresas como la deseabilidad de este comportamiento dependerían de la viabilidad percibida, mientras que esta última dependería de la influencia que tienen las normas sociales asociadas al contexto familiar. Por consiguiente, el modelo generado muestra una compleja relación entre el emprendimiento y la influencia familiar en los emprendedores potenciales tal y como señalaron, por ejemplo, Stanworth et al. (1989) y Rogoff y Lee (1996). Así, en la medida en que la familia valore positivamente la creación de empresas propias, los hijos tenderán a decantarse por esta alternativa laboral y esto, según el modelo generado, podría explicar la viabilidad, la deseabilidad, la influencia social de los grupos de iguales y la actitud hacia la creación de empresas propias. Es particularmente interesante que el modelo TAN haya identificado un camino dirigido desde la influencia familiar hasta la actitud hacia la creación de empresas dado que, como señalaba Allport (1935) en el siglo pasado, uno de los cuatro pilares fundamentales de la creación de actitudes proviene de la influencia parental.
El algoritmo de descubrimiento estructural automático también sugiere que el locus de control es una variable mediadora entre los obstáculos y la viabilidad percibida frente a la creación de una empresa. Esta relación tiene sentido desde un punto de vista teórico desde que se sabe que el locus de control interno es un rasgo que se asocia con las personas emprendedoras (e.g., Sánchez, 2003). Sin embargo, la relación que se establece entre influencia normativa social, locus de control e intención emprendedora parece ser más compleja de lo que se pensaba con anterioridad. Por ejemplo, la influencia de las normas sociales sobre la intención emprendedora, así como otras variables asociadas a la creación de empresas, parece ser más indirecta de lo que se creía anteriormente (e.g, Heuer y Liñán, 2013). En este sentido Siu y Lo (2013) han observado recientemente que la influencia de las normas sociales con relación a la intención emprendedora depende del grado en que una sociedad puede considerarse más colectivista o más individualista.

Por su parte, los resultados de Zellweger, Sieger y Halter (2011) contrastan con la observación clásica que indica que el locus de control se asocia a la intención emprendedora. No obstante, tal y como indican en su trabajo, la relación entre locus de control e intención emprendedora podría estar mediada por la influencia familiar, de modo que los descen-

$$
\text { Revista de Psicología }
$$$$
\text { 2014, 23(2), 71-87 }
$$ 
dientes de familias emprendedoras "se dan cuenta de que la capacidad de controlar su propio destino al optar por una carrera emprendedora puede ser mayormente una ilusión" (Zellweger et al., 2011, p. 531) si tenemos en cuenta el volumen de responsabilidades y presiones a la que están sometidos estos profesionales. Nuestros resultados arrojan luz sobre la compleja relación que podría existir entre estas variables; futuras investigaciones podrán poner a prueba hipótesis relacionadas con las mismas.

Adicionalmente, el modelo TAN establece relaciones entre los diferentes componentes del riesgo y los diferentes formatos en los que se pueden entender la viabilidad de la creación de empresas que convendría estudiar en futuras investigaciones. Ello podría ser de utilidad, como hemos indicado más arriba, frente a la orientación de emprendedores potenciales con el ánimo de que evalúen sensatamente sus prospectivas de éxito en sus propuestas de negocio. De este modo, podrían reducir sensiblemente sus probabilidades de fracaso debidas a una mala gestión de sus creencias hacia los riesgos que tienen que asumir a lo largo del proceso (e.g., Cooper et al., 1988; Hmieleski y Baron, 2009; Lovallo y Kahneman, 2003). Por último, cabe mencionar el desfase que se observa en la capacidad predictiva del modelo TAN y su poder predictivo. Dado que el aumento que se produce en la bondad de ajuste de este modelo es marginal con relación a los otros dos y considerando que se reduce sensiblemente la parsimonia del modelo (en términos de número de parámetros y enlaces), futuros trabajos tendrían que profundizar en este aspecto para desarrollar modelos más aceptables desde el punto de vista metodológico. El uso y comparación de diferentes tipos de algoritmos de aprendizaje estructural podría ser una estrategia apropiada en este sentido.

Para terminar, es conveniente destacar algunas limitaciones al trabajo realizado, así como futuras líneas de investigación que pudiesen arrojar luz sobre el fenómeno del emprendimiento y que pudiesen servir de cara a la mejora del desarrollo social de nuestro entorno cercano. En primer lugar, sería deseable desarrollar estudios análogos a este en los que se utilizase una muestra de emprendedores reales, con el objetivo de comparar los resultados y las conclusiones que se han presentado en este trabajo. Esto podría servir para tratar de identificar diferencias y similitudes entre un perfil y otro con el ánimo de ahondar en la comprensión del fenómeno emprendedor. También sería deseable realizar más estudios destinados a clarificar las relaciones que el algoritmo TAN ha identificado entre las variables analizadas para tratar de ahondar en el entendimiento de la compleja relación que se establece entre las variables utilizadas. Como han intentado recientemente Ruiz-Ruano, López y Scutari (2014), utilizar otros algoritmos de búsqueda estructural podría ser de utilidad en este sentido. Desde el punto de vista metodológico podríamos criticar la generalización de nuestros resultados, dado el reducido tamaño muestral. Aunque, como hemos indicado anteriormente, el tamaño de muestra es un problema que las redes bayesianas parecen tolerar bien, un estudio con mayor tamaño muestral podría aportar evidencias sobre la validez cruzada del modelo estimado.

A modo de conclusión, creemos que la potenciación de las actitudes emprendedoras tendría que enfocarse a la incipiente área del emprendimiento sostenible. Esta forma de emprendimiento está orientada a la búsqueda simultánea de beneficio económico, social y medioambiental (e.g., Dean y McMullen, 2007; López, 2012; Shepherd y Patzelt, 2011) y cada vez está recibiendo más atención en el ámbito de la educación superior (e.g., Jones, Selby y Sterling, 2010; Springett, 2010). Así, en cierto modo, podremos aspirar a favorecer el desarrollo del sistema autorrenovable del que hablaba Shapero (1981) hace más de tres décadas apoyándonos en el sistema de enseñanza universitario (Wheeler et al., 2005). 


\section{Referencias}

Ajzen, I. y Fishbein, M. (1980). Understanding attitudes and predicting social behavior. Englewood, NJ: Prentice-Hall.

Ajzen, I. y Fishbein, M. (2005). The influence of attitudes on behavior. En D. Albarracín, B. T. Hohnson y M. P. Zanna (Eds.), The handbook of attitudes (pp. 173-221). Mahwah, NJ: Erlbaum

Allport, G. W. (1935). Attitudes. En C. Murchison (Ed.), Handbook of social psychology (pp. 798-844). Worcester, MA: Clark University Press.

Anderson, R. D. y Vastag, G. (2004). Causal modeling alternatives in operations research: Overview and applications. European Journal of Operational Research, 156, 92-109.

Bhave, M. P. (1994). A process model of entreprenuerial venture creation. Journal of Business Venturing, 9, 223-242.

Bird, B. (1988). Implementing entrepreneurial ideas: The case for intention. Academy of Management Review, 13, 442-453.

http://dx.doi.org/10.5465/AMR.1988.4306970

Block, J. H., Hoogerheide, L. F. y Thurik, A. R. (2012). Are education and entrepreneurial income endogenous? A Bayesian analysis. Entrepreneurship Research Journal, 2(3), 1-29. http://dx.doi.org/10.1515/2157-5665.1051

Boyatzis, R. E. (1982). Competence at work. En A. J. Stewart (Ed.), Motivation and society (pp. 221-243). San Francisco, CA: JosseyBass.

Cantillon, R. (2010). An essay on economic theory. Auburn, AL: Ludwig von Mises Institute. (Trabajo original publicado en 1755).

Cohen, B. y Winn, M. I. (2007). Market imperfections, opportunity and sustainable entrepreneurship. Journal of Business Venturing, 22, 29-49.

http://dx.doi.org/10.1016/j.jbusvent.2004.12.001
Cooper, A. C., Woo, C. Y. y Dunkelberg, W. C. (1988). Entrepreneurs' perceived chances for success. Journal of Business Venturing, 3, $97-108$. http://dx.doi.org/10.1016/0883-9026(88)90020-1

Cowell, R. G., Dawid, A. P., Lauritzen, S. L. y Spiegelhalter, D. J. (1999). Probabilistic networks and expert systems. Harrisonburg, VA: Springer.

Dean, T. J. y McMullen, J. S. (2007). Towards a theory of sustainable entrepreneurship: Reducing environmental degradation through entrepreneurial action. Journal of Business Venturing, 22, 50-76.

http://dx.doi.org/10.1016/j.jbusvent.2005.09.003

Dreiler, P., Blenker, P. y Nielsen, K. (2003). Promoting entrepreneurship - changing attitudes or behavior? Journal of Small Business and Enterprise Development, 10, 383-392. http://dx.doi.org/10.1108/14626000310504693

Edwards, W. (1998). Hailfinder. Tools for and experiences with Bayesian normative modeling. American Psychologist, 53, 416-428. http://dx.doi.org/10.1037/0003-066X.53.4.416

Edwards, W. y Fasolo, B. (2001). Decision technology. Annual Review of Psychology, 52, 581-606.

Fishbein, M. y Ajzen, I. (1975). Belief, attitude, intention, and behavior: An introduction to theory and research. Reading, MA: Addison-Wesley.

Fitzsimmons, J. R. y Douglas, E. J. (2011). Interaction between feasibility and desirability in the formation of entrepreneurial intentions. Journal of Business Venturing, 26, 431-440. http://dx.doi.org/10.1016/j.jbusvent.2010.01.001

Fuller-Love, N. (2006). Management development in small firms. International Journal of Management Reviews, 8(3), 175-190. http://dx.doi.org/10.1111/j.14682370.2006.00125. $\mathrm{x}$ 
García, J., Cano, C. J. y Gea, A. B. (2005). Actitudes emprendedoras en estudiantes universitarios y empresarios. Evidencias de validez de un instrumento. Iberpsicología, 10(8), art. 12.

Gartner, W. B. (1985). A conceptual framework for describing the phenomenon of new venture creation. Academy of Management Review, 10, 696-706.

http://dx.doi.org/10.2307/258039

Gartner, W. B. (1988). "Who is an entrepreneur?" is the wrong question. American Journal of Small Business, 12(4), 11-32.

Genescá, E. y Capelleras, J. L. (2004). Un análisis comparativo de las características de las microempresas en España. Universia Business Review, 2, 72-93.

Greiner R., Su, X., Shen, B. y Zhou, W. (2005). Structural extension to logistic regression: Discriminative parameter learning of belief net classifiers. Machine Learning, 59, 297-322. http://dx.doi.org/10.1007/s10994-0050469-0

Greiner R. y Zhou, W. (2002). Structural extension to logistic regression: Discriminative parameter learning of belief net classifiers. En R. Dechter, M. Kearns y R. Sutton (Eds.), Proceedings of the Eighteenth Annual National Conference on Artificial Intelligence (pp. 167-173). Palo Alto, California: American Association for Artificial Intelligence.

Heckerman, D. (1995). A tutorial on learning with Bayesian networks (Rep. Téc. MS-TR-95-06). Redmon, WA: Microsoft Research.

Heuer, A. y Liñán, A. (2013). Testing alternative measures of subjective norms in entrepreneurial intention models. International Journal of Entrepreneurship and Small Business, 19, 35-50.

http://dx.doi.org/10.1504/IJESB.2013.054310

Hmieleski, K. M. y Baron, R. A. (2009). Entrepreneurs' optimism and new venture performance: A social cognitive perspective. Academy of Management Journal, 52, 473-488. http://dx.doi.org/10.5465/AMJ.2009.41330755
Jones, P., Selby, D. y Sterling, S. (2010). Introduction. En P. Jones, D. Selby y S. Sterling (Eds.), Sustainability education. Perspectives and practice across higher education (pp. 116). Londres: Earthscan.

Krueger, N. F. y Brazeal, D. V. (1994). Entre preneurial potential and potential entrepreneurs. Entrepreneurship Theory and Practice, 18, 91-104.

Krueger, N. F. y Carsrud, A. L. (1993). Entrepreneurial intentions: Applying the theory of planned behavior. Entrepreneurship and Regional Development, 5, 315-330.

http://dx.doi.org/10.1080/089856293000 00020

Krueger, N. F., Reilly, M. D. y Carsrud, A. L. (2000). Competing models of entrepreneurial intentions. Journal of Business Venturing, 15, 411-432.

http://dx.doi.org/10.1016/S0883-

9026(98)00033-0

Liang, C. y Dunn. P. (2008). Are entrepreneurs optimistic, realistic, both or fuzzy? Relationships between entrepreneurial traits and entrepreneurial learning. Academy of Entrepreneurship Journal, 14, 51-73.

Liang, C. y Dunn. P. (2010). Entrepreneurial characteristics, optimism, pessimism, and realism - correlation or collision? Journal of Business and Entrepreneurship, 22, 1-22.

Licht, A. N. y Siegel, J. I. (2006). The social dimensions of entrepreneurship. En M. Casson, B. Yeung, A. Basu y N. Wadeson (Eds.), The Oxford handbook of entrepreneurship (pp. 514-539). Nueva York: Oxford University Press.

LimeSurvey Project Team. (2013). LimeSurvey [Aplicación de software libre y de código abierto para desarrollar encuestas online]. Recuperado de https://www.limesurvey.org/es

Liñán, F., Battistelli, A. y Moriano, J. A. (2008). Entrepreneurial intentions in Europe. En J. A. Moriano, M. Gorgievski, y M. Lukes 
(Eds.), Teaching psychology of entrepreneurship: Perspectives from six European countries (pp. 21-43). Madrid: UNED.

Liñán, F., Rodríguez-Cohard, J. C. y RuedaCantuche, J. M. (2011). Factors affecting entrepreneurial intention levels: A role for education. International Entrepreneurship Management Journal, 7, 195-218. http://dx.doi.org/10.1007/s11365-010-0154z

López, J. (2012). Modelos actitudinales y emprendimiento sostenible. Cuaderno Interdisciplinar de Economía Sostenible, 8, 111-131.

López, J. y García, J. (2011a). Utilidad de las redes bayesianas en psicología. Almería: Editorial de la Universidad de Almería.

López, J. y García, J. (2011b). Eventos por variable en regresión logística y redes bayesianas para predecir actitudes emprendedoras. Revista Electrónica de Metodología Aplicada, 16, 43-58.

López, J. y García, J. (2012). Comparative study on entrepreneurial attitudes modelled with logistic regression and Bayes nets. The Spanish Journal of Psychology, 15, 1147-1162. http://dx.doi.org/10.5209/rev_SJOP.201 2.v15.n3.39404

López, J., Ramírez, A. y Casado, M. P. (2012). Modelling entrepreneurial attitudes in women entrepreneurs with Bayesian networks. Psychology, 3, 265-271. http://dx.doi.org/10.4236/psych.2012.33037

Lovallo, D. y Kahneman, D. (2003). Delusions of success. How optimism undermines executives' decisions. Harvard Business Review, 81, 56-63.

Martínez, I. y Rodríguez, C. (2003). Modelos gráficos. En Y. del Águila et al. (Eds.), Técnicas estadísticas aplicadas al análisis de datos (pp. 217-257). Almería: Servicio de Publicaciones de la Universidad de Almería.

McClelland, D. (1955). Some social consequences of achievement motivation. En M. R. Jones (Ed.), Nebraska symposium on motivation. Lincoln, NE: University of $\mathrm{Ne}-$ braska Press.

McClelland, D. (1961). The achieving society. New York: Free Press.

McKenzie, B., Ugbah, S. y Smothers, N. (2007). "Who is an entrepreneur" is still the wrong question? Academy of Entrepreneurship Journal, 13, 23-43.

McMullen, J. S. y Shepherd, D. A. (2006). Entrepreneurial action and the role of uncertainty in the theory of the entrepreneur. Academy of Management Review, 31, 132-152. http://dx.doi.org/10.5465/AMR.2006.19 379628

Moriano, J. A., Gómez, A., Laguna, M. y Roznowski, B. (2008). Validación de un cuestionario para medir la intención emprendedora. Una aplicación en España \& Polonia. En J. F. Morales, C. Huici, A. Gómez y E. Gaviria (Coords.), Método, teoría e investigación en psicologia social (pp. 101-121). Madrid: Pearson.

Nadkarni, S. y Shenoy, P. P. (2004). A causal mapping approach to constructing Bayesian networks. Decision Support Systems, 38, 259281.

http://dx.doi.org/10.1016/S01679236(03)00095-2

Neapolitan, R. E. (1990). Probabilistic reasoning in expert systems: Theory and algorithms. New York: Wiley \& Sons.

Norsys Software Corp. (2013). Netica [A complete software to work with Bayesian belief networks, decision nets and influence diagrams]. Recuperado de http://norsys.com

Pearl, J. (1978). An economic basis for certain methods of evaluating probabilistic forecasts. International Journal of Man-Machine Studies, 10, 175-183.

http://dx.doi.org/10.1016/S0020-

7373(78)80010-8

R Core Team. (2014). R [A language and envi- 
ronment for statistical computing]. Recuperado de

http://www.R-project.org

Robinson, P. B., Stimpson, D. V., Huefner, J. C. y Hunt, H. K. (1991). An attitude approach to the prediction of entrepreneurship. Entrepreneurship Theory and Practice, 15, 13-31.

Rogoff, E. G. y Lee, M. S. (1996). Does firm origin matter? An empirical examination of types of small business owners and entrepreneurs. Academy of Entrepreneurship Journal, 1, 1-17.

Ruiz-Ruano, A. M., López, J. y Scutari, M. (2014). Learning a Bayesian structure to model attitudes towards business creation at university. En L. Gómez, A. López y I. Candel (Eds.), INTED2014 Proceedings (pp. 5242-5249). Valencia: International Association of Technology, Education and Development.

Sánchez, M. L. (2003). El perfil psicológico del autoempleado. (Tesis doctoral, Universidad Complutense de Madrid, Madrid, España). Recuperado de http://eprints.ucm.es/tesis/psi/ucmt26888.pdf

Schlaegel, C. y Koenig, M. (2014). Determinants of entrepreneurial intent: A metaanalytic test and integration of competing models. Entrepreneurship Theory and Practice, 38, 291-332.

http://dx.doi.org/10.1111/etap.12087

Shapero, A. (1975). The displaced, uncomfortable entrepreneur. Psychology Today, 9, 83-133.

Shapero, A. (1981). Entrepreneurship. Key to self-renewing economies. Economic Development Commentary, 5, 19-23.

Shapero, A. (1985). Why entrepreneurship? A worldwide perspective. Journal of Small Business Management, 23(4), 1-5.

Shapero, A. y Sokol, L. (1982). The social dimensions of entrepreneurship. En C. Kent, D. Sexton, y K. H. Vesper (Eds.),
The encyclopedia of entrepreneurship (pp. 72 90). Englewood Cliffs: Prentice-Hall.

Shen, B., Su, X., Greiner, R., Musilek, P. y Cheng, C. (noviembre, 2003). Discriminative parameter learning of general Bayesian network classifiers. Comunicación presentada en el 15th IEEE International Conference on Tools with Artificial Intelligence (ICTAI-03). Sacramento.

Shepherd, D. A. y Patzelt, H. (2011). The new field of sustainable entrepreneurship: Studying Entrepreneurial Action Linking "what is to be sustained" with "what is to be developed". Entrepreneurship Theory and Practice, 35, 137-173.

http://dx.doi.org/10.1111/j.1540-

6520.2010.00426.x

Siu, W. y Lo, E. S. (2013). Cultural contingency in the cognitive model of entrepreneurial intention. Entrepreneurship Theory and Practice, 37, 147-173.

http://dx.doi.org/10.1111/j.1540-

6520.2011.00462.x

Smilor, R. W. y Feeser, H. R. (1991). Chaos and the entrepreneurial process: Patterns and policy implications for technology entrepreneurship. Journal of Business Venturing, 6 , 165-172.

Sohn, S. Y. y Lee, A. S. (2013). Bayesian network analysis for the dynamic prediction of early stage entrepreneurial activity index. Expert Systems with Applications, 40, 4003-4009.

http://dx.doi.org/10.1016/j.eswa.2013.01 .009

Springett, J. F. (2010). Education for sustainability in the business studies curriculum: Ideological struggle. En P. Jones, D. Selby y S. Sterling (Eds.), Sustainability education. Perspectives and practice across higher education (pp. 75-92). Londres: Earthscan.

Stanworth, J., Stanworth, C., Granger, B. y Blyth, S. (1989). Who becomes an entrepreneur? International Small Business Journal, 8, 11-22. 
http://dx.doi.org/10.1177/026624268900 800101

Thompson, J. L. (2004). The facets of the entrepreneur: Identifying entrepreneurial potential. Management Decision, 42, 243-258.

Veciana, J. M. (1989). Características del empresario en España. Papeles de Economía Española, 39, 19-36.

Welsch, H. P. y Young, E. C. (1982). The information source selection decision: The role of entrepreneurial personality characteristics. Journal of Small Business Management, 20, 49-57.
Wheeler, D., McKague, K., Thomsom, J., Davies, R., Melaye, J. y Prada, M. (2005). Creating sustainable local enterprise networks. MIT Sloan Management Review, 47, 33-40.

Zellweger, T., Sieger, P. y Halter, F. (2011). Should I stay or should I go? Career choice intentions of students with family business background. Journal of Business Venturing, 26, 521-536.

http://dx.doi.org/10.1016/j.jbusvent.201 0.04 .001

Fecha de recepción: 4 de diciembre de 2014

Fecha de aceptación: 19 de diciembre de 2014 